\title{
Practical Stimulus Design for a Multi-Tone Fit
}

\author{
Marko Neitola
}

\begin{abstract}
A multi-tone measurement can be used to characterize the noise and distortion of a device or a system. Such assessment can be accomplished by a least-squares sinusoidal fit, which is widely analyzed in the prior art. The IEEE standard sinusoidal least-squares fit (sinefit) can be quite easily expanded to multiple tones, real or complex. From a designer point-ofview, the challenge is to generate a proper stimulus. The design questions in such case are the following: "How do I design the tone spacing?", "What is the minimum record length of the stimulus with n tones?", "Can I use the 3-parameter fit (known frequencies)?", "How many iterations do I need in the 4-parameter fit (unknown frequencies)?" and "How accurate the initial guess of the frequencies have to be for the 4-parameter fit?". This study strives to answer the aforementioned questions with reflections to the analytical results from prior art.
\end{abstract}

Index Terms-Measurement, Characterization, IEEE sinefit

\section{INTRODUCTION}

A least-squares fit of a sinusoid can be used to characterize the distortion and noise of various devices and systems. The fitting algorithms have been standardized in the IEEE standard 1057 [1] and IEEE standard 1241 [2]. The 3-parameter fit finds the amplitude, phase and offset for a known frequency. For unknown frequency (typical case), the 4-parameter sinefit finds the amplitude, phase, offset and frequency iteratively.

To characterize a device or a system by a multi-tone fit requires designing an array of stimulus with proper length $\mathrm{N}$ and frequency separation. This work concentrates mainly on the typical usage of tone fitting i.e. 4-parameter multi-tone fit, in which the initial guess for the tone frequencies may have some uncertainty.

In [3], Händel and Zetterberg expanded the single-tone sinusoid fit to complex domain (cisoid fit) and proposed a least squares approach to determine the I/Q imbalance of a direct-conversion receiver. Their transformation to complex domain was realized in polar form and the tone frequency was found by a built-in Matlab function. In Section II, the IEEE standard sinefit is introduced and also generalized for complex-domain tones (cisoids) in both rectangular and polar form. The basis functions and the transformation between rectangular and polar form are described in Sect. II.

In [4], Anderson and Händel studied the least-squares multitone fit in real domain. They used the IEEE standard sinefit [1] in combination with the Expectation-Maximization (EM) algorithm [5] to improve the parameter estimation. In [6], Anderson and Händel introduced the generalized version of the IEEE sinefit procedure for real-domain multi-tones. The idea in [6] was to combine all necessary basis functions for each

The author is with the CAS research unit, Faculty of Information Technology and Electrical Engineering, University of Oulu, Finland, e-mail: marko.neitola@oulu.fi. tone into a single observation matrix. This is also used here and the generalization further refined in Sect. II by expanding the multitone fit into complex domain.

Section III demonstrates fit examples with identical noise floor but with different bandwidths. As will be shown in the later Sections, bandwidth contributes to fit errors such as magnitude, phase and frequency, but the residual variance $\hat{\sigma}^{2}$ should remain constrained for a successful fit in a Gaussian noise environment. The requirement for the bandwidthindependent $\hat{\sigma}^{2}$ will be used to investigate the often debatable question on 4-parameter sinefit: When to stop the iterative fitting algorithm? This condition is typically determined by providing a tolerance on minimal parameter change during iterations or a by a fixed number of iterations.

Section IV suggests the pivotal figures of merit in the fit: the frequency difference and initial error normalized by bandwidth and Fourier resolution. This type of normalization enables expressing the number of samples as a function of bandwidth, number of tones and the normalized frequency difference.

In [7], Anderson and Händel derived asymptotic CramérRao lower bounds (CRBs) for three and four model parameters under the Gaussian assumption. They provided the expression on the maximum initial frequency error for the 3-parameter sinefit, which will be expanded to equidistant multi-tones in V. Section V also demonstrates that the initial error boundary for successful 4-parameter fit is quite insensitive to bandwidth.

Section VI presents the lower limits for the bandwidthindependent Fourier-normalized frequency difference, which defines the lower limit for the multi-tone data record length. It will be shown that such limit is dependent on how the tones are spaced and whether the multi-tone data is real or complex. Analytical limit suggested by prior art is reached only by a complex multi-tone. Fit error estimates are also presented and reflected to the prior art.

\section{BACKGROUND}

In the sinefit [1], [2], a real sinusoid $y(t)$ is perceived as in (1), where the a and $b$ define the sinusoid magnitude and phase and c defines the dc-offset. For known frequency $\omega$, the the observation matrix $\mathbf{H}$ is formed by the basis functions, i.e. the multiplicands of $\{a, b, c\}:\{\cos (\omega t), \sin (\omega t), 1\}$. The size of the observation matrix $\mathbf{H}$ is $\mathrm{N}$-by-3, where $\mathrm{N}$ is the number of samples in the data array $\mathbf{y}$. The parameter estimates are obtained by the least squares fit (3), where the results in $\mathbf{X}$ are the estimates $[\hat{a}, \hat{b}, \hat{c}]^{T}$. 


$$
\begin{array}{rc}
y(t, \omega)= & a \cdot \cos (\omega t)+b \cdot \sin (\omega t)+c \\
& \{a, b, c\} \in \mathbf{R} \\
\mathbf{y}= & {\left[y_{1}, y_{2}, \cdots y_{N}\right]^{T}} \\
\mathbf{X}= & \left(\mathbf{H}^{T} \mathbf{H}\right)^{-1} \mathbf{H}^{T} \mathbf{y}
\end{array}
$$

The sinusoid fit is typically iterative where the estimated frequency $\hat{\omega}$ is refined in few iterations. In the IEEE standard 4-parameter sinusoid fit, the iterative fit is based on the basis function from the $2^{\text {nd }}$ order Taylor series expansion with respect to the frequency error $\widetilde{\omega}(4)$. For the real sinusoid with offset, the parameter $\alpha$ in the Taylor expansion (5) is presented in (6). The observation matrix of the 4-parameter sinefit $(\mathbf{H})$ has therefore four columns to accommodate the frequency error $\widetilde{\omega}$. The 4-parameter sinefit is iterative i.e. updates the values of $\mathbf{X}=[\hat{a}, \hat{b}, \hat{c}, \hat{\omega}]^{T}$ extracted from the previous iteration.

$$
\begin{array}{rlc}
\omega & = & \hat{\omega}+\widetilde{\omega} \\
y(t, \omega) & \approx & y(t, \hat{\omega})+\widetilde{\omega} \cdot \alpha(t, \hat{\omega}) \\
\alpha(t, \hat{\omega}) & = & t \cdot(b \cdot \cos (\hat{\omega} t)-a \cdot \sin (\hat{\omega} t))
\end{array}
$$

To expand the IEEE standard sinusoid fit into complex domain is very straightforward. A rectangular-form cisoid defined in (7) and its Taylor expansion (8)-(9) are structurally similar to the real counterparts (5)-(6), respectively. The fitted estimates of A and B are complex.

$$
\begin{array}{cc}
y_{C R}(t, \omega)= & A \cdot \cos (\omega t)+j B \cdot \sin (\omega t)+C \\
& \{A, B, C\} \in \mathbf{C}, j=\sqrt{-1} \\
y(t, \hat{\omega}) \approx & y(t, \hat{\omega})+\alpha_{C R}(t, \hat{\omega}) \\
\alpha_{C R}(t, \hat{\omega})= & t \cdot(j \cdot B \cdot \cos (\hat{\omega} t)-A \cdot \sin (\hat{\omega} t))
\end{array}
$$

Another way to perform the 4-parameter cisoid fit is to use the polar form (10) and its Taylor expansion in (11) and (12).

$$
\begin{array}{cc}
y_{C P}(t, \omega)= & D \cdot \exp (j \omega t)+E \cdot \exp (-j \omega t)+F \\
& \{D, E, F\} \in \mathbf{C} \\
y_{C P}(t, \hat{\omega}) \approx & y_{C P}(t, \hat{\omega})+\alpha_{C P}(t, \hat{\omega}) \\
\alpha_{C P}(t, \hat{\omega})= & +j t \cdot(D \cdot \exp (j \hat{\omega} t)-E \cdot \exp (-j \hat{\omega} t))
\end{array}
$$

Using the the polar form in the cisoid fit provides the estimates of fundamental (D) and image (E) magnitude and phase directly. The transformation between the two forms can be found by trigonometric recursion formula, resulting in the conversion formulas (14)-(15).

$$
\begin{array}{r}
C=F \\
A=D+E \text { and } B=D-E \\
D=0.5 \cdot(A+B) \text { and } E=0.5 \cdot(A-B)
\end{array}
$$

A cisoid fit in polar form is fully equivalent to the fit in the rectangular form, i.e. the fit results are identical. Moreover, the least squares fit to rectangular or polar form with 3 or 4 parameters is in-line with the real least-squares fit as the fit will cover both positive and negative frequencies (which is also the case in real sinefit).

As the cisoid may contain noise and distortion, the frequency error result from the least-squares fit $\widetilde{\omega}$ may turn complex and result in unsuccessful convergence in the iterative 4-parameter fit. To avoid this, the imaginary part of the observation matrix should be omitted.

To expand the least-squares sinefit or cisoid fit to cover multiple tones is simply adding more columns into the observation matrix. For $\mathrm{N}$-sized data vector $\mathrm{y}$ containing $\mathrm{n}$ tones, the size of the 3-parameter observation matrix $\mathbf{H}$ would be N-by- $(1+2 n)$ (offset, $n$ fundamentals and $n$ images). For 4-parameter multi-tone fit, the matrix size would be N-by$(1+3 n)$, where the extra column is for the estimates of the frequency error.

From a multi-tone fit, there are several figures of merit to assess the fit quality. From the fit residual (17), one can estimate e.g. intermodulation distortion or simply the residual variance (18). The latter is an important merit when studying the fit solely with added Gaussian noise. The estimated variance $\hat{\sigma}^{2}$ should be quite close to the real variance $\hat{\sigma}^{2}$.

$$
\begin{aligned}
\hat{\mathbf{y}} & =\mathbf{X} \cdot \mathbf{H} \\
\widetilde{\mathbf{y}} & =\mathbf{y}-\hat{\mathbf{y}} \\
\hat{\sigma}^{2} & =\operatorname{var}(\widetilde{\mathbf{y}})
\end{aligned}
$$

The actual fit errors are presented in (22)-(24). Here (and throughout this paper), it is assumed that the fundamental and image tones (and their estimates) are represented as arrays as in (19) and (20), respectively. In (22), $\oslash$ denotes element-wise division.

As the focus in this paper will be on the 4-parameter fit, the frequency estimates in the array $\hat{\mathbf{w}}$ will contain error $\widetilde{\mathbf{w}}$ (21). The frequency error from (23) is normalized by $2 \pi \cdot f_{s}$, where $f_{s}$ is the sampling frequency.

$$
\begin{array}{rlrl}
\mathbf{D}= & & {\left[D_{1}, D_{2}, \cdots, D_{n}\right]^{T}} \\
\mathbf{E}= & & {\left[E_{1}, E_{2}, \cdots, E_{n}\right]^{T}} \\
\mathbf{w}= & & \left.\omega_{1}, \omega_{2}, \cdots, \omega_{n}\right]^{T}=2 \pi \mathbf{f}=\hat{\mathbf{w}}+\widetilde{\mathbf{w}} \\
|\widetilde{\mathbf{D}}|= & & (|\mathbf{D}|-|\hat{\mathbf{D}}|) \oslash|\mathbf{D}| \\
\widetilde{\mathbf{f}}= & & (\mathbf{f}-\hat{\mathbf{f}}) / f_{s}=(\mathbf{w}-\hat{\mathbf{w}}) /\left(2 \pi \cdot f_{s}\right) \\
\angle \widetilde{\mathbf{D}}= & & & \angle \mathbf{D}-\angle \hat{\mathbf{D}}
\end{array}
$$

The complex multi-tone fit in the polar form enables a direct way to assess IQ-imbalance. For a known magnitude imbalance $\Gamma(d B)$ and phase skew $\theta$ (degrees/radians), the expected image response rejection ratio (IMRR) can be calculated by (26) [9]. From the multi-tone fit, the estimate of IMRR can be obtained from $\hat{\mathbf{D}}$ and $\hat{\mathbf{E}}$ by (27). 


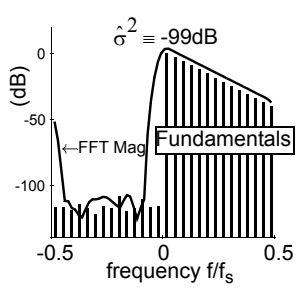

(a)

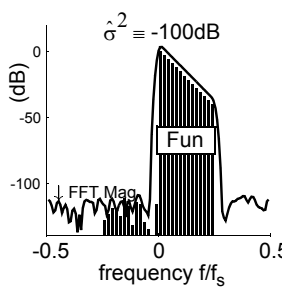

(b)

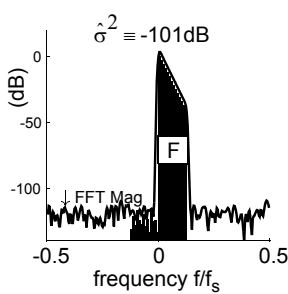

(c)
Fig. 1. 4-parameter sinefit (7 iterations) for 14-tone cisoid fit with Gaussian noise $\sigma^{2}=-100 d B$ : a) $\mathrm{sps}=1$, b) $\mathrm{sps}=2$ and c) $\mathrm{sps}=4$.

$$
\begin{aligned}
& \gamma=\quad 10^{\Gamma / 20} \\
& \operatorname{IMRR}=\frac{\left(\gamma^{2}+1-2 \gamma \cdot \cos (\theta)\right)}{\left(\gamma^{2}+1+2 \gamma \cdot \cos (\theta)\right)} \\
& \mathrm{IMRR}=\frac{\hat{\mathbf{E}}^{H} \hat{\mathbf{E}}}{\hat{\mathbf{D}}^{H} \hat{\mathbf{D}}}
\end{aligned}
$$

\section{Minimum Number of Iterations}

The challenging part of fitting multiple sinusoids or cisoids is to declare an n-tone stimulus whose (often noisy and distorted) system response can be fitted. The examples in Fig. 1 are cisoid $n$-tones with $n=14$. In Fig. 1, the frequency spacing is equidistant and the bandwidth in (28) is defined by the sampling frequency $f_{s}$ and samples per symbol i.e. sps (28).

$$
B W=\frac{f_{s}}{2 \cdot \operatorname{sps}} H z
$$

Figure 1 displays the fit residual variance $\hat{\sigma}^{2}(18)$, which is in-line with the added Gaussian noise $\sigma^{2}=-100 \mathrm{~dB}$. The fit residual (17) represents the noisy and/or distorted part of the multi-tone 4-parameter fit (with a small initial frequency error). A non-ideal fit also contains some fit error which is proportional to the added noise. As can be seen from Fig. 1, there are image tones below the $-100 \mathrm{~dB}$ noise floor: the added Gaussian noise restricts the accuracy of the tone frequencies.

The number of samples $\mathrm{N}$ is $65 \cdot \mathrm{sps}$ in Fig. 1. As the tone spacing relative to $\mathrm{N} / \mathrm{sps}$ remains constant in Figures 1a-1c, doubling or quadrupling $\mathrm{N}$ has very little difference in residual variances $\hat{\sigma}^{2}$. In other words, the variances $\hat{\sigma}^{2}$ are independent on the bandwidth.

Increasing $\mathrm{N}$ by the multiplicand sps decreases the fit errors (22)-(24), which are further discussed in Sect. VI. Proper tone spacing is further discussed in Sect. IV.

For the multi-tone fit results shown in Fig. 1, the number if iterations is fixed to 7. The proper condition of 4-parameter sinefit convergence has many opinions in the literature. The IEEE standard [1], [2] proposes stopping the iteration, when the maximum change in $[\hat{a}, \hat{b}, \hat{c}]$ is lower than a predefined tolerance TOL (29). Another version is to stop the iterations when the change in $\hat{\omega}$ is lower than TOL (30) [3]. Also a fixed number of 4 iterations has bee proposed in [4] (31).

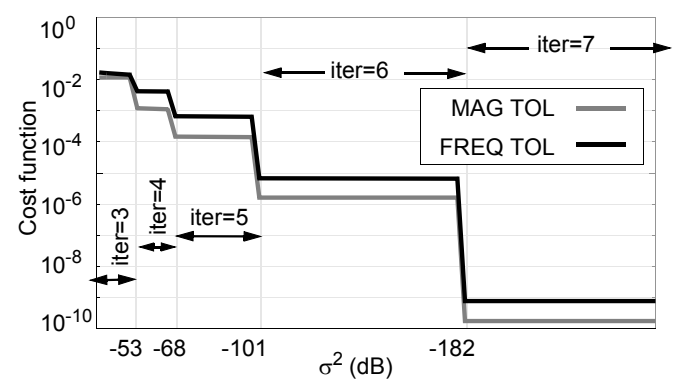

Fig. 2. Sweep of sps with maximum $\hat{\sigma}^{2}$ deviation limited to $<3 \mathrm{~dB}$ : the error based on classic tolerance limits: magnitude (29) and frequency (30).

$$
\begin{gathered}
\left|\max \left(\left[\hat{a_{i}}, \hat{b_{i}}, \hat{c_{i}}\right]-\left[\hat{a}_{i-1}, \hat{b}_{i-1}, \hat{c}_{i-1}\right]\right)\right|<\mathrm{TOL} \\
\left|\widetilde{\omega}_{i}-\widetilde{\omega}_{i-1}\right|<\mathrm{TOL} \\
\text { iter }=4
\end{gathered}
$$

Let's sweep Gaussian noise for the examples in Fig. 1. In the following $\sigma^{2}$ sweep, three cases of sps $\in[1,2,4]$ are fitted initially by two iterations. If the three values of $\hat{\sigma}^{2}$ differ by more than $3 \mathrm{~dB}$, the number of iterations is incremented until all $\hat{\sigma}^{2}$ are within the $3 \mathrm{~dB}$ range.

The common convergence conditions (cost functions) in (30) or (29) are illustrated in Fig. 2. The stairs-like curves represent the actual tolerance limits that would result in the displayed number of iterations. As the bandwidth independence requirement forces the residual variances to vary less than $3 \mathrm{~dB}$, the number of iterations is clearly inversely dependent on $\sigma^{2}$. Without a priori information on $\sigma^{2}$, the cost functions (30) and (29) are quite useless.

The observations from Fig. 2 favor using fixed number of iterations. In a practical system response measurement result, the noise floor level of the measured data is typically $\sigma^{2}>$ $-100 d B$, where the number of iterations can be fixed to e.g. 5 . As the cost of extra iterations is in the range of milliseconds, it would be prudent to add a safety margin to the number of iterations.

\section{Multi-Tone Spacing}

The tone spacing can be either linear or nonlinear, i.e. equidistant or non-equidistant. The minimum frequency difference (33) normalized to Fourier resolution i.e. bin, is presented in (34): $\operatorname{bin}_{\mathrm{Nyq}}=1$ is the Fourier (or Rayleigh) resolution. In addition to Fourier-normalization, (34) is also bandwidthnormalized, hence the subscript "Nyq". For instance, halving the bandwidth doubles $\mathrm{N}$ and halves $\Delta f_{\min }$.

$$
\begin{array}{rcc}
\Delta \omega_{\text {min }} & = & 2 \pi \Delta f_{\text {min }} \\
\Delta f_{\text {min }} & = & \min \left(\left|f_{i}-f_{i-1}\right|\right), i=\{2,3, \ldots, n\} \\
\operatorname{bin}_{\mathrm{Nyq}} & = & N \cdot \frac{\Delta f_{\text {min }}}{f_{s}}
\end{array}
$$

\section{A. Equidistant Tones}

For equidistant (linearly spaced) tone spacing, the bandwidth-independent tone-spacing parameter in (34) can be 
presented as a function of $\mathrm{n}$ (the number of tones). From such parameter, the number of samples can be expressed. Here, the fundamental frequencies are assumed to have the same sign to accommodate both real and complex multi-tones. A complex fundamental cisoids may have both negative and positive frequencies, which is discussed in Sect. IV-B

Let's define an auxiliary $n$-sized array $\mathbf{k}$ in (35). The intended tone frequencies in the array $\mathbf{f}$ are generated by $\mathbf{k}$ in (37). Equidistant tone spacing is a common strategy in generating an n-tone stimulus. Linearly spaced array $\mathbf{k}$ is defined in (36), where parameter $\kappa$ in (41) defines the step $\Delta k$.

For the optimal equidistant tones $(n>1)$, the frequency difference normalized to Fourier resolution i.e. bin can be expressed as (38). Normalized to Nyquist band, the corresponding Fourier resolution is (39).

$$
\begin{array}{rcc}
\mathbf{k} & = & \left.\left[k_{1}, k_{2}, \cdots, k_{n}\right]^{T} \in\right] 0,1[ \\
& = & {\left[\kappa, \kappa+\Delta_{k}, \kappa+2 \Delta_{k}, \cdots 1-\kappa\right]^{T}} \\
\mathbf{f} & = & \mathbf{k} \cdot \frac{f_{s}}{2 \cdot \mathrm{sps}}(\mathrm{Hz}) \\
\operatorname{bin}= & \Delta k \cdot \frac{N}{2} \\
\operatorname{bin}_{\mathrm{Nyq}} & = & \Delta k \cdot \frac{N}{2 \cdot \mathrm{sps}}
\end{array}
$$

From (39), the minimum number of samples $\mathrm{N}$ that satisfies the parameter bin can therefore be expressed as in (40).

$$
N=\operatorname{sps} \cdot\left\lceil\frac{\operatorname{bin}_{\mathrm{Nyq}}}{\Delta k}\right\rceil
$$

To reach minimum $\mathrm{N}$ in (44), the value of $\kappa$ in (41) ensures that the minimum frequency difference between fundamental and image tones is equal to $\Delta k$ in (42). The bandwidth- and Fourier-normalized frequency difference and the number of samples will be (43) and (44).

$$
\begin{array}{rc}
\kappa & =\frac{0.5}{n} \\
\Delta k & =\frac{1}{n} \\
\operatorname{bin}_{\mathrm{Nyq}}= & \frac{N}{2 n \cdot \mathrm{sps}} \\
N= & \mathrm{sps} \cdot\left\lceil\mathrm{bin}_{\mathrm{Nyq}} \cdot 2 n\right\rceil
\end{array}
$$

For a multi-tone, the linearly spaced tone frequencies defined by (41) and (42) result in the minimal number of samples for given $\left\{\mathrm{n}, \mathrm{sps}, \mathrm{bin}_{\mathrm{Nyq}}\right\}$. However, if the inter-modulation distortion (IMD) is the merit of interest, some issues need to be considered.

For a complex multi-tone defined in full Nyquist band $(\mathrm{sps}=1)$ and $\kappa$ in $(41)$ results in IMD tones exactly at the image frequencies. In an ideal case, this would result in IMD magnitudes and phases directly in the image fit result $\mathbf{E}$ (20). This may seem appealing, but there are two disadvantages. Firstly, if the measurement system contributes dynamic error, some IMD tones may not be fitted as image frequencies.

Secondly, it can be deduced from [7], that the fit error magnitude at $\omega_{i}$ is proportional to the magnitude at $-\omega_{i}$ (and vice versa). Basically, the image tones contains some distortion from the fit itself, so it may be prudent to separate IMD tones from image tones.

To ensure that the IMD tones will not be fitted as image tones, the following procedure shifts the frequency spacing. For the linearly spaced vector $\mathbf{k}$ in (36), the parameter dimensioning $\{\kappa, \Delta k\}$ can be represented as (45)-(47). In (45), $\lambda=2$ results in (41) and (42). For $0<\lambda<2$, the minimal distance between image and fundamental is larger than the fundamental frequency difference. As a result, IMD tones will not appear at image or fundamental frequencies.

$$
\begin{array}{rcc}
\beta & = & \left.\left.\frac{n}{2+\lambda \cdot(n-1)}, \lambda \in\right] 0,2\right] \\
\kappa & = & \beta / n \\
\Delta k & = & \frac{(1-2 \beta) / n}{n-1}
\end{array}
$$

For an example, if $\lambda=1, \kappa$ and $\Delta k$ in (36) would be (48). The tone spacing based on (48) results in the number of samples presented in (49), i.e. the increase for the required number of samples is quite small compared to (44).

$$
\begin{array}{rcc}
\kappa=\Delta k= & \frac{1}{n+1} \text { for } \lambda=1 \\
N= & \text { sps } \cdot\left\lceil\text { bin }_{\mathrm{Nyq}} \cdot 2(n+1)\right\rceil \text { for } \lambda=1
\end{array}
$$

\section{B. Other Types of Multi-Tone Stimuli}

For sps $>1$, the equidistant frequencies in (37) can be shifted from baseband to e.g. $f_{s} / 4$ without any changes to tone spacing or minimum required number of samples. Moreover, the recommended minimum $\operatorname{bin}_{\mathrm{Nyq}}$ for various $\mathrm{n}$ suggested Sect. VI are unaffected by the center frequency shift.

A complex multi-tone can have both negative and positive fundamental frequencies. If the fundamental tones cover the both positive and negative frequencies within the bandwidth, the image tones can be placed between fundamental tones by converting (41) and (42) by (50) and (51). As a result, the required number of samples remains the same as in (44), because the tone spacing in a cluster of fundamental and image tones will be defined by (42).

$$
\begin{array}{rc}
\mathbf{k} \rightarrow & 2 \mathbf{k}-1-\kappa \\
\Delta k \rightarrow & 2 \Delta k
\end{array}
$$

If the n-tone (real or complex) has non-equidistant tones, the minimum difference in the non-linearly spaced array $\mathbf{k}$ defines $\Delta k$ in (40). Moreover, $\Delta k$ between fundamental and image tones should be verified.

\section{UNCERTAINTy OF THE INITIAL GUESS}

The initial error of the tone frequency $\widetilde{\omega}_{0}$ can be expressed as a function of normalized to Fourier resolution, see (52). From (52), the normalized initial frequency error bin err $_{\text {can }}$ be expressed as in (53).

Here, $\widetilde{\omega}_{0}$ (and bin $_{\text {err }}$ ) should be perceived as the maximum absolute initial frequency error. In the behavioral studies performed in this study, the added error has only the two 
extreme values $\pm \widetilde{\omega}_{0}$, where the sign of the error is uniformly distributed. As a consequence, the range of initial guess error is $2 \cdot$ bin $_{\text {err }}$.

$$
\begin{aligned}
\widetilde{\omega}_{0}= & 2 \pi \cdot \widetilde{f}_{0}=2 \pi \cdot f_{s} \cdot \operatorname{bin}_{\mathrm{err}} / N \\
\operatorname{bin}_{\mathrm{err}}= & \frac{\widetilde{\omega}_{0} \cdot N}{2 \pi \cdot f_{s}}
\end{aligned}
$$

The 3-parameter fit relies on known frequencies. The term "known" can be translated to a tiny error proportional to the noise variance $\sigma^{2}$. Increasing $\sigma^{2}$ naturally decreases the accuracy of the fit.

In [7], Andersson and Händel suggested the initial frequency error upper boundary (54), under which the 3-parameter sinefit should be favored (with Gaussian noise assumption). The right-hand side of (54) is exactly the Cramér-Rao lower bound on the parameter $\omega$ [7].

Let's expand (54) to complex domain. The expansion to complex rectangular and polar forms (for single-tone) are shown in (55) and (56), respectively. The polar form in (56) was created by the conversion rule (14).

$$
\begin{aligned}
\widetilde{\omega}_{0}^{2} & =\frac{24 \sigma^{2}}{\left(a^{2}+b^{2}\right) N^{3}} \\
\widetilde{\omega}_{0}^{2} & =\frac{24 \sigma^{2}}{\left(A^{H} A+B^{H} B\right) N^{3}} \\
\widetilde{\omega}_{0}^{2} & =\frac{12 \sigma^{2}}{\left(D^{H} D+E^{H} E\right) N^{3}}
\end{aligned}
$$

Replacing $\widetilde{\omega}_{0}$ in (56) by (52) results in (57). Replacing $\mathrm{N}$ by (44) results in the boundary of bin err as a function of multitone parameters $\mathrm{n}$ and bin $_{\mathrm{Nyq}}$.

$$
\begin{aligned}
&\left(2 \pi \cdot f_{s} \cdot \operatorname{bin}_{\mathrm{err}} / N\right)^{2}= \\
& \operatorname{bin}_{\mathrm{err}}^{2}=\frac{12 \sigma^{2}}{\left(D^{\prime} D+E^{\prime} E\right) N^{3}} \\
& \operatorname{sps} \cdot 2 n \pi^{2} \cdot \operatorname{bin}_{\mathrm{Nyq}} \cdot\left(D^{\prime} D+E^{\prime} E\right)
\end{aligned}
$$

The analytical boundary for bin err is visualized in Fig. 3. In Fig. $3 \mathrm{a}$, the number of tones $\mathrm{n}$ and the Gaussian noise variance $\sigma^{2}$ are swept with fixed $\operatorname{bin}_{\mathrm{Nyq}}=2.5$. For logarithmically decreasing noise variance, the boundary for bin $n_{\text {err }}$ decreases proportionally. It is logical to conclude that using a 3-parameter fit may prove unfeasible if the n-tone contains any measurement noise.

In Fig. $3 b, \sigma^{2}$ is fixed to the level of $-50 \mathrm{~dB}$ and $\left\{n, \operatorname{bin}_{\mathrm{Nyq}}\right\}$ are swept. Again, the the lower bound of bin err $_{\text {is quite tiny. }}$

To demonstrate how bin $_{\text {err }}$ and bandwidth (term sps) affects the multi-tone fit success of a 4-parameter fit, Fig. 4 shows how the frequency error (23) changes as a function of $n$ and bin $_{\text {err }}$ at full, half and quarter Nyquist bandwidth in Fig. 4a $4 c$, respectively. Here, $\operatorname{bin}_{\mathrm{Nyq}}$ is fixed to 2.5 and the number of tones $\mathrm{n}$ is swept from 2 to 31 .

All three types of fit errors in (22)-(24) were also assessed similarly as in Fig. 4 and with different values of $\sigma^{2}$. The different errors have different dynamics, but the boundary of successful fit for all three types of error (22)-(24) are quite similar at fixed $\left\{\right.$ bin $\left._{\mathrm{Nyq}}, \mathrm{sps}\right\}$. In other words, increasing $\sigma^{2}$ changes fit the error magnitudes, but not the successboundaries.

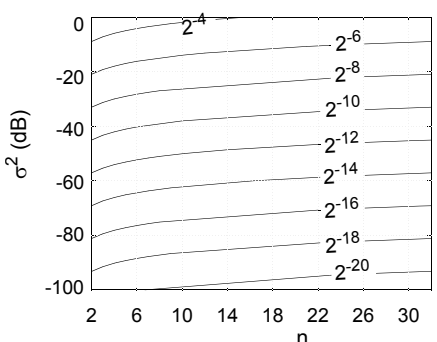

(a)

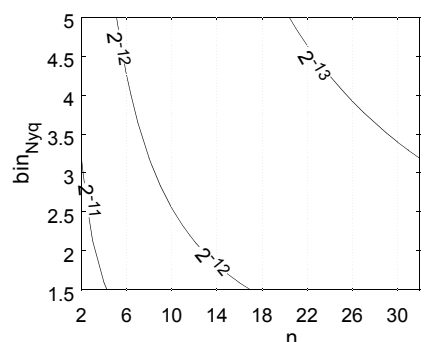

(b)

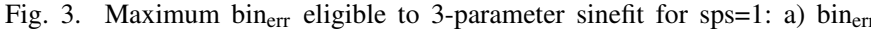
versus $\left\{\sigma^{2}, n\right\}$ with fixed bin Nyq $=3$ and b) bin err versus $\left\{\operatorname{bin}_{\mathrm{Nyq}}, n\right\}$ with fixed $\sigma^{2} \equiv-50 \mathrm{~dB}$.
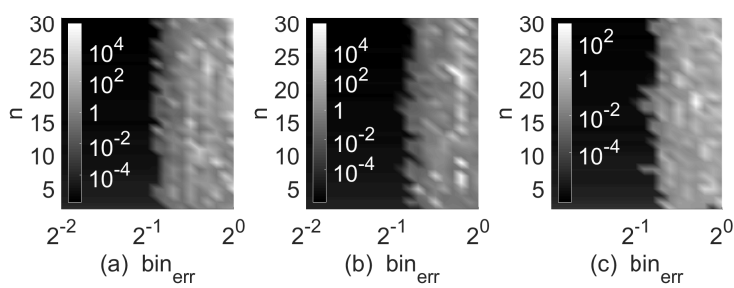

Fig. 4. Frequency error (23) versus $\left\{n\right.$, $\left.\operatorname{bin}_{\mathrm{err}}\right\}$ at $\sigma^{2}=-50 \mathrm{~dB}$ : a) sps $=1$ (Nyquist bandwidth), b) $\mathrm{sps}=2$ and c) $\operatorname{sps}=4$.

According to Fig. 4, the maximum bin err $_{\text {for }}$ all $\mathrm{n}$ is around half of Fourier resolution. This will be further specified in the next Section.

\section{Practical Tone Spacing Limits of the 4-PARAmeter Multi-Tone Fit}

In [6], Anderson and Händel discussed the performance of multi-tone methods as follows: "If detection and initialization are performed in a correct and successful way the error variance of the overall method is expected to be close to the Cramér-Rao bound (CRB). If the spectral components are well separated in frequency each parameter estimate is expected to reach its corresponding $\mathrm{CRB}$, that for the frequency approximately coincides with the single tone CRB in (59). However, if two sinewaves are closely located in frequency the single tone assumption is not valid. In [8], it is shown that if the frequency separation $\Delta \omega$ is larger than about 1.5 times the Fourier resolution then the CRB in the dual tone case basically coincides with (59)." [6]

$$
\operatorname{Var}(\hat{\omega}) \geq \frac{24 \sigma^{2}}{\left(a_{i}^{2}+b_{i}^{2}\right) \cdot N}, N \rightarrow \infty, i \in[1,2]
$$

Here, the goal is to find $\operatorname{bin}_{\mathrm{Nyq}}$ for a 4-parameter fit that guarantees $\hat{\sigma}^{2} \approx \sigma^{2}$ with any bandwidth for multi-tones with added Gaussian noise. The number of 4-parameter fit iterations was fixed to 9 .

In the following test, the normalized frequency error bin $_{\mathrm{err}}$, sps, $\mathrm{n}$ and the noise variance $\sigma^{2}$ are swept to find values of $\operatorname{bin}_{\mathrm{Nyq}}$ in (43) that ensure that the fit error residual $\hat{\sigma}^{2}$ is independent on bandwidth (term sps). The test has the following seven steps for a certain values of $\left\{n, \operatorname{bin}_{\mathrm{err}}, \sigma^{2}\right\}$ :

1) Set $\operatorname{bin}_{\mathrm{Nyq}}$ to 1 


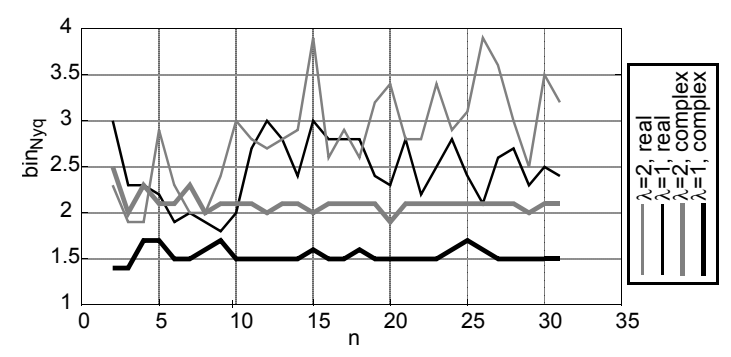

Fig. 5. Lower limit for the bandwidth independent $\operatorname{bin}_{\mathrm{Nyq}}$ for $n \in[2,31]$.

2) For $\operatorname{sps} \in\{1,2,4,8\}$ (28), tabulate all $\hat{\sigma}^{2}$ from n-tone fits.

3) From the four sps-values, record the maximum deviation of between all $\hat{\sigma}^{2}$.

4) If the maximum deviation is $>3 \mathrm{~dB}$, increase bin $_{\mathrm{Nyq}}$ by 0.1 and go to step 2 .

5) If the absolute deviation is $\leq 3 \mathrm{~dB}$, the bandwidthindependence is fulfilled.

The above test was conducted for real and complex domain and $\lambda \in\{1,2\}$ (45). The following parameters were swept:

- $n \in[2,3,4, \cdots, 31]$.

- bin $_{\text {err }} \in 2^{-X}$, where $X \in[-6, \cdots,-0.5]$, step-size 0.25 .

- $\sigma^{2} \in[-300, \cdots, 0] \mathrm{dB}$, step-size $20 \mathrm{~dB}$.

The following observation was made for each case of $\{n, \lambda$, real/complex $\}$ : bin $_{N y q}$ is a constant within $-240 d B<\sigma^{2}<$ $-60 d B$ and bin $_{\text {err }}<2^{-1.5}$. The constant value $\operatorname{bin}_{\mathrm{Nyq}}$ depends heavily on $\lambda$ and whether the multi-tone is real or complex, as can be seen from Fig. 5. According to Fig. 5, increasing $n$ changes the lower limit of bin $_{\mathrm{Nyq}}$, especially in the case of real multi-tone.

For the real multi-tones in Fig. 5, the lower limit of bin $_{\mathrm{Nyq}}$ is higher compared with the complex case. This is due to the fact that the two-sided spectrum of a real sinusoid has an image tone with the fundamental magnitude. As was assumed in Sect. IV-A, the magnitudes at $\{\omega,-\omega\}$ contribute to each other's fit error. Such fit-induced distortion explains the higher values of bin $_{\mathrm{Nyq}}$.

For the complex multi-tones and $\lambda=2$, bin $_{\mathrm{Nyq}}$ is higher than in the case of $\lambda=1$. Even though the results presented in Fig. 5 do not contain nonlinear distortion (only Gaussian noise), the fit-inflicted tones are statistically larger at $\lambda=2$, compared with $\lambda=1$.

The lowest bin $_{\mathrm{Nyq}}$ limits can be obtained with complex multi-tones and $\lambda=1$, where the safe lower limit in Gaussian environment is $\operatorname{bin}_{\mathrm{Nyq}} \approx 1.7$. This is quite close to the analytical prediction of 1.5 [8] (ref. [6]).

The maximum absolute fit error based on (22)-(24) with sps $\in[1,2,4,8]$ can be approximated from the sweep results and fitting the worst-case absolute error versus $\left\{\sigma^{2}\right.$, sps $\}$. Fit errors versus the $\left\{\sigma^{2}, \mathrm{sps}\right\}$ are nearly a flat surface in the logarithmic scale of all 3 dimensions, so the fit errors can be approximated via a linear two-dimensional least-squares fit.

The estimates for complex case and $\lambda=1$ are shown in (60)-(62). The parameter values (multiplicands and exponen- tials) are averaged from parameters obtained from $n \in[8,31]$. The value of $N_{\text {Nyq }}$ is calculated by the $\operatorname{bin}_{\mathrm{Nyq}}$ values in Fig. 5 .

$$
\begin{array}{rc}
\max (|\widetilde{f}|) & \approx \quad 1.3 \sqrt{\frac{\left.\left(\sigma^{2}\right)^{1.0}\right)}{N_{\mathrm{Nyq}}^{3} \cdot \mathrm{sp}^{3.0}}} \\
\max (|\widetilde{D}|) & \approx \quad 2.4 \sqrt{\frac{\left.\left(\sigma^{2}\right)^{1.0}\right)}{N_{\mathrm{Nyq}} \cdot \mathrm{sps}^{1.0}}} \\
\max (|\angle \widetilde{D}|) & \approx \quad 170 \sqrt{\frac{\left.\left(\sigma^{2}\right)^{1.0}\right)}{N_{\mathrm{Nyq}} \cdot \mathrm{sps}^{0.9}}}(\mathrm{deg})
\end{array}
$$

The two-dimensioal fit results are the multiplicand and the exponents of $\sigma^{2}$ and sps in (60)-(62). The exponents in (60)(62) are very well in-line with the proportionals based on Cramér-Rao lower bound on the error variances presented in [7]: $\operatorname{Var}(\widetilde{f}) \propto \frac{\sigma^{2}}{N^{3}}, \operatorname{Var}(|\widetilde{D}|) \propto \frac{\sigma^{2}}{N}$ and $\operatorname{Var}(\angle \widetilde{D}) \propto \frac{\sigma^{2}}{N}$.

\section{CONCLUSIONS}

The focus on this work is on how to optimize a multi-tone stimulus so that the response can be fitted. The IEEE standard sinefit [1], [2] was here expanded to real and complex multitones without any additional algorithms. The functions used in this work are available in [10].

To generate a proper multi-tone stimulus, this work suggests using tone frequency parameters that are independent on the number of tones and bandwidth.

For a real or complex multi-tone with $\mathrm{n}$ tones, the bandwidth-independent tone-spacing parameters were here used to find:

- The expression of number of samples as a function of $n$.

- The analytical upper boundary of the frequency error in 3-parameter fit based on [7].

- The tone spacing for a reliable 4-parameter fit result.

- The upper limit for initial frequency errors.

- The distortion contribution of a 4-parameter fit.

- The coarse maximal fit error expressions (magnitude, phase, frequency), which share the proportionals of analytical results in [7].

\section{REFERENCES}

[1] "IEEE Standard for digitizing waveform recorders," IEEE Std. 1057, 1994.

[2] "IEEE Standard for Terminology and Test Methods for Analog-toDigital Converters," IEEE Standard 1241, 2000.

[3] P. Händel and P. Zetterberg "Receiver I/Q Imbalance: Tone Test, Sensitivity Analysis, and the Universal Software Radio Peripheral, " in IEEE Trans. Instrumentation and Measurement, Vol. 59, No. 3, March 2010.

[4] T. Anderson and P. Händel "Multiple-Tone Estimation by IEEE Standard 1057 and the Expectation-Maximization Algorithm, " in IEEE Trans. Instrumentation and Measurement, Vol. 54, No. 5, Oct. 2005.

[5] A. Dempster and N. Laird and D. Rubin, "Maximum likelihood from incomplete data via the EM algorithm," in j. Roy. Stat. Soc. B, vol. 39, no. 1 , pp. 1-38, 1977.

[6] T. Anderson and P. Händel "Toward a standardized multi-sinewave fit algorithm," in Proc. 9th Eur. Workshop ADC Modelling Testing, Athens,Greece, Sep. 2001, pp. 337-342.

[7] T. Anderson and P. Händel "IEEE Standard 1057, Cramér-Rao Bound and the Parsimony Principle, " in IEEE Trans. Instrumentation and Measurement, Vol. 55, No. 1, Feb. 2006 
[8] D. N. Swingler "Further simple approximations to the Cramér-Rao lower bound on frequency estimates for closely-spaced sinusoids," IEEE Trans. Acoust., Speech, Signal Process., vol. 43, no. 1, pp. 367-369, Jan. 1995.

[9] J. K. Cavers and M. W. Liao, "Adaptive compensation for imbalance and offset losses in direct conversion transceivers," in IEEE Trans. Vehicular Technology, Vol 42, No. 4, Nov 1993.

[10] M. Neitola (2019). Sinefit for multitones

https://www.mathworks.com/matlabcentral/fileexchange/72382-sinefitfor-multitones, MATLAB Central File Exchange. Retrieved August 7, 2019. 